APPENDIX TABLE DR1: FORAMINIFERAL DISTRIBUTION DATA FOR CORE 1098B.

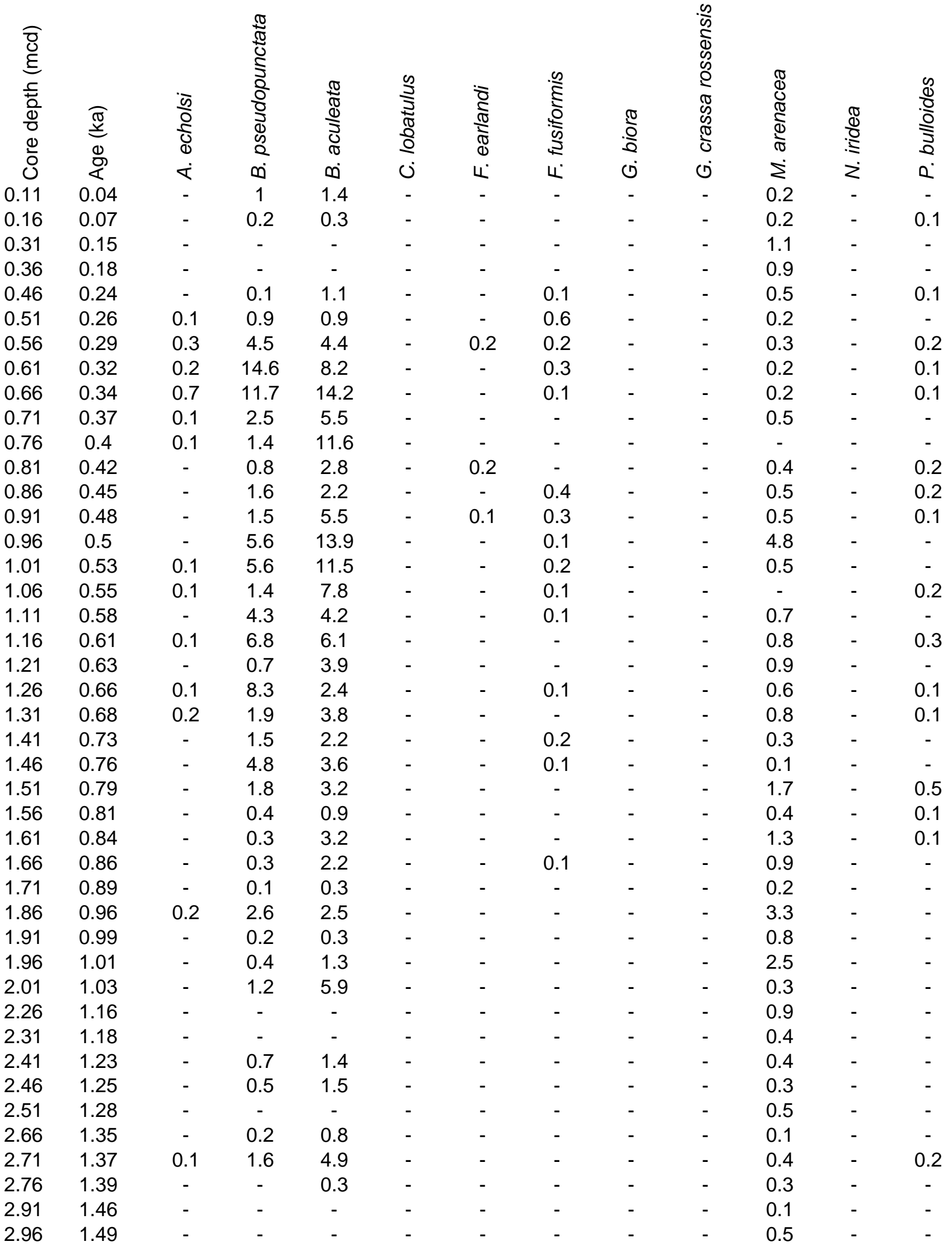


APPENDIX TABLE DR1: FORAMINIFERAL DISTRIBUTION DATA FOR CORE 1098B.

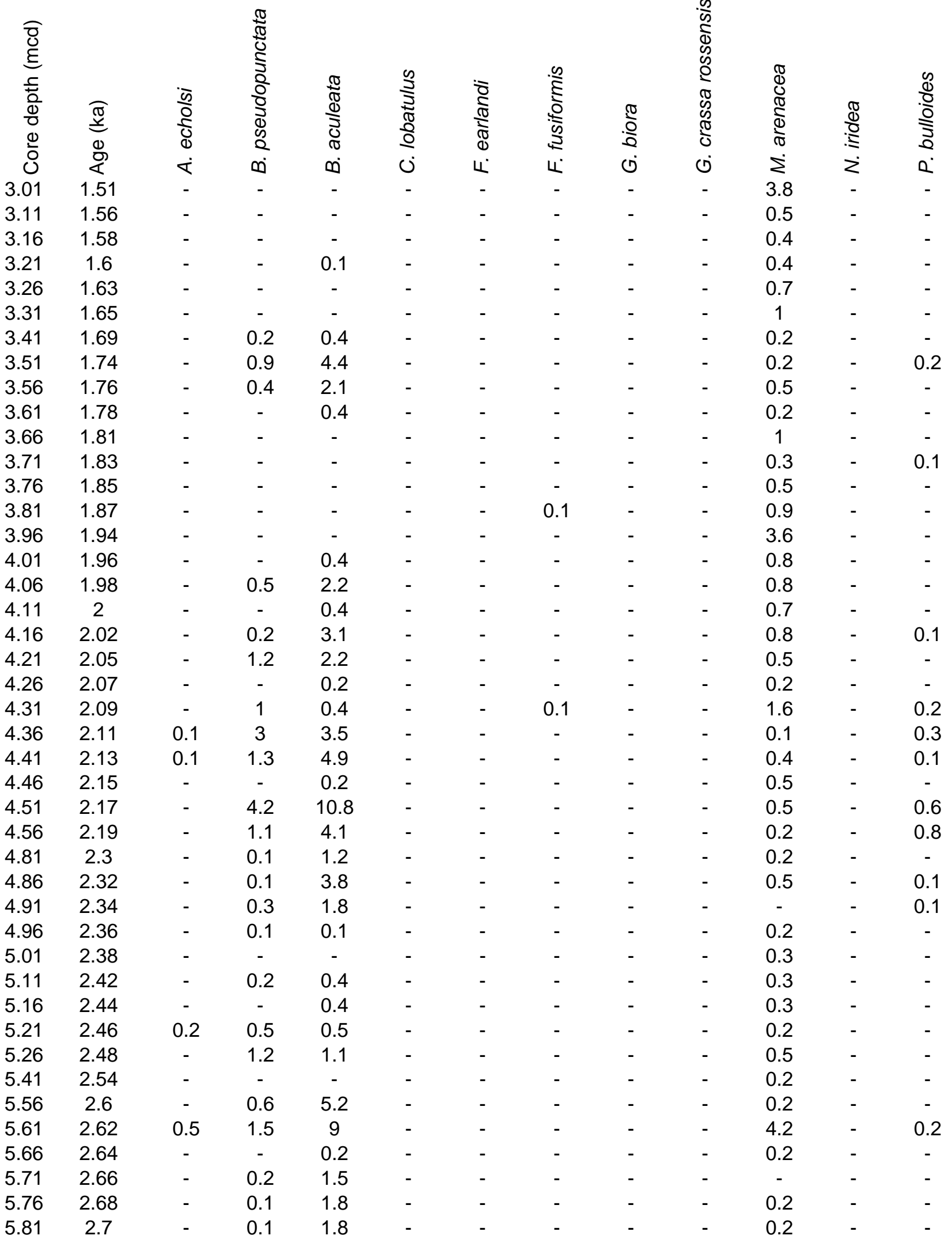


APPENDIX TABLE DR1: FORAMINIFERAL DISTRIBUTION DATA FOR CORE 1098B.

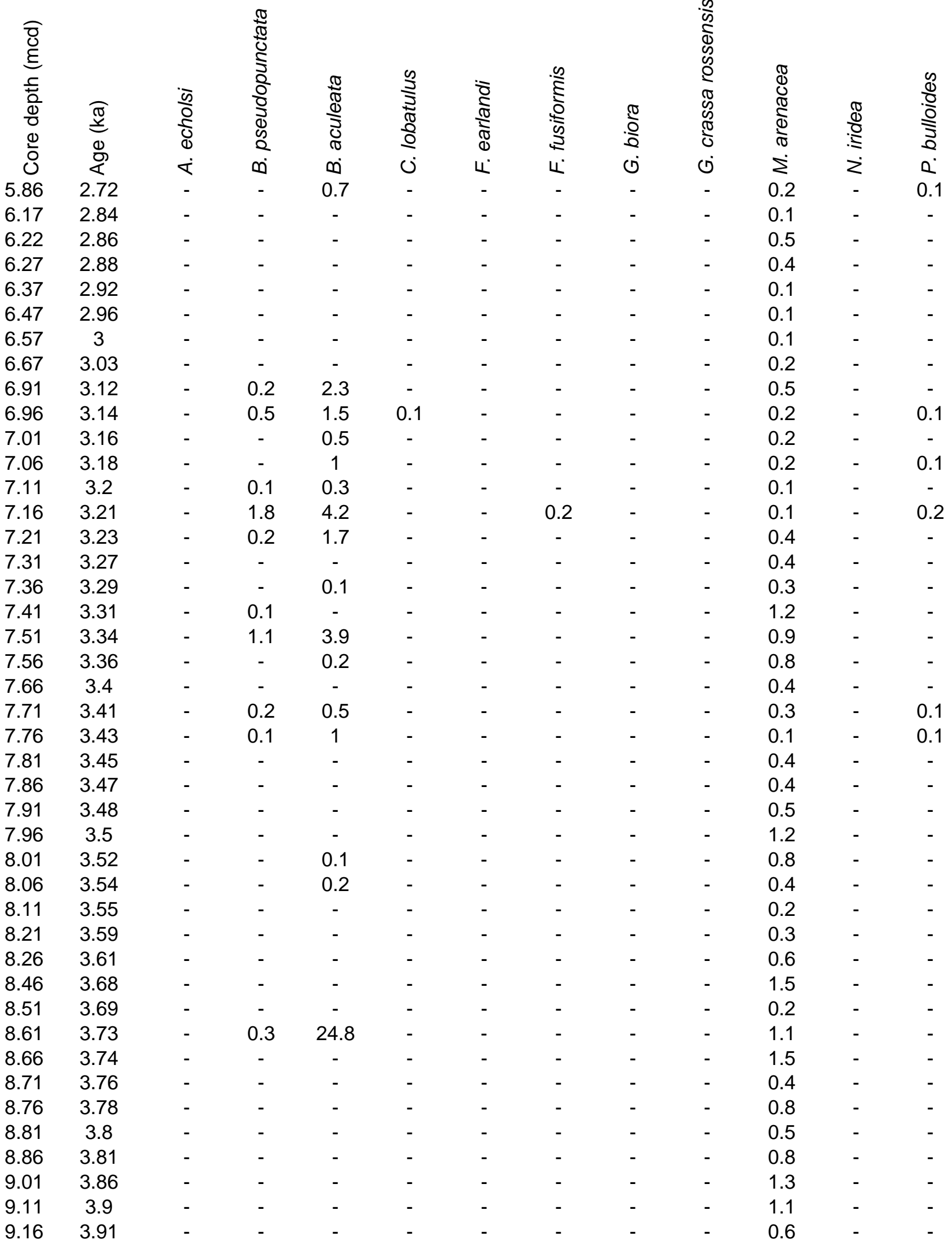


APPENDIX TABLE DR1: FORAMINIFERAL DISTRIBUTION DATA FOR CORE 1098B.

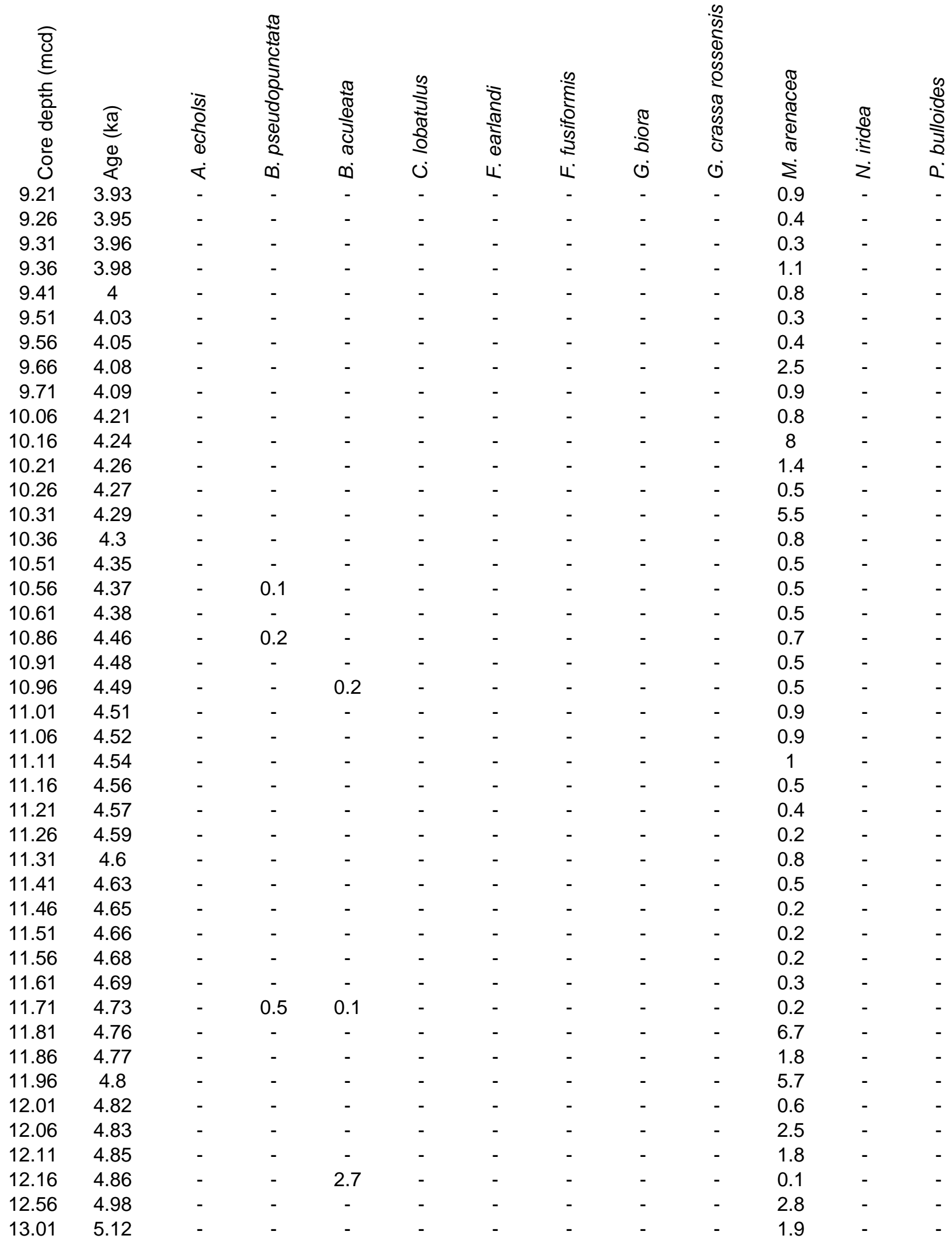


APPENDIX TABLE DR1: FORAMINIFERAL DISTRIBUTION DATA FOR CORE 1098B.

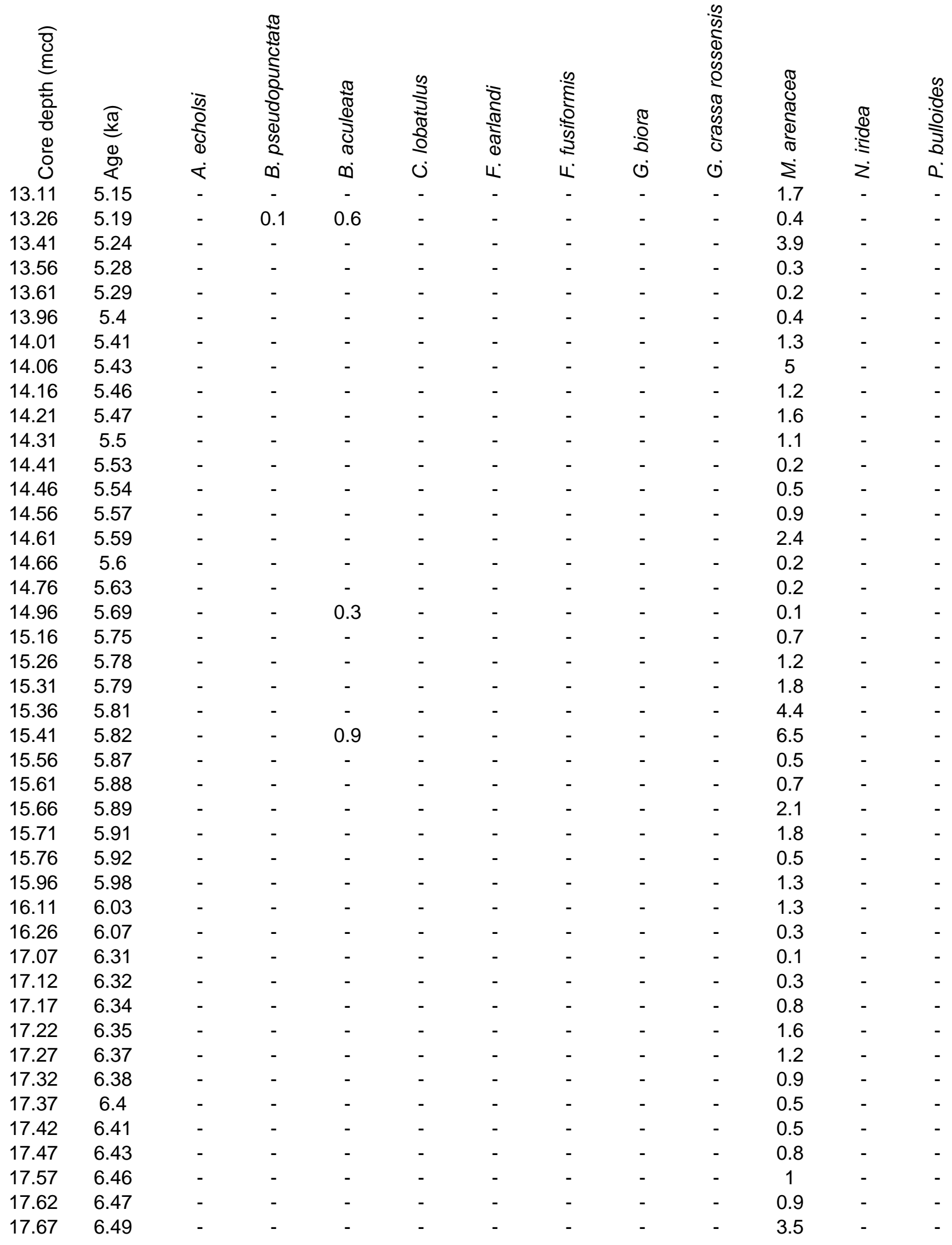


APPENDIX TABLE DR1: FORAMINIFERAL DISTRIBUTION DATA FOR CORE 1098B.

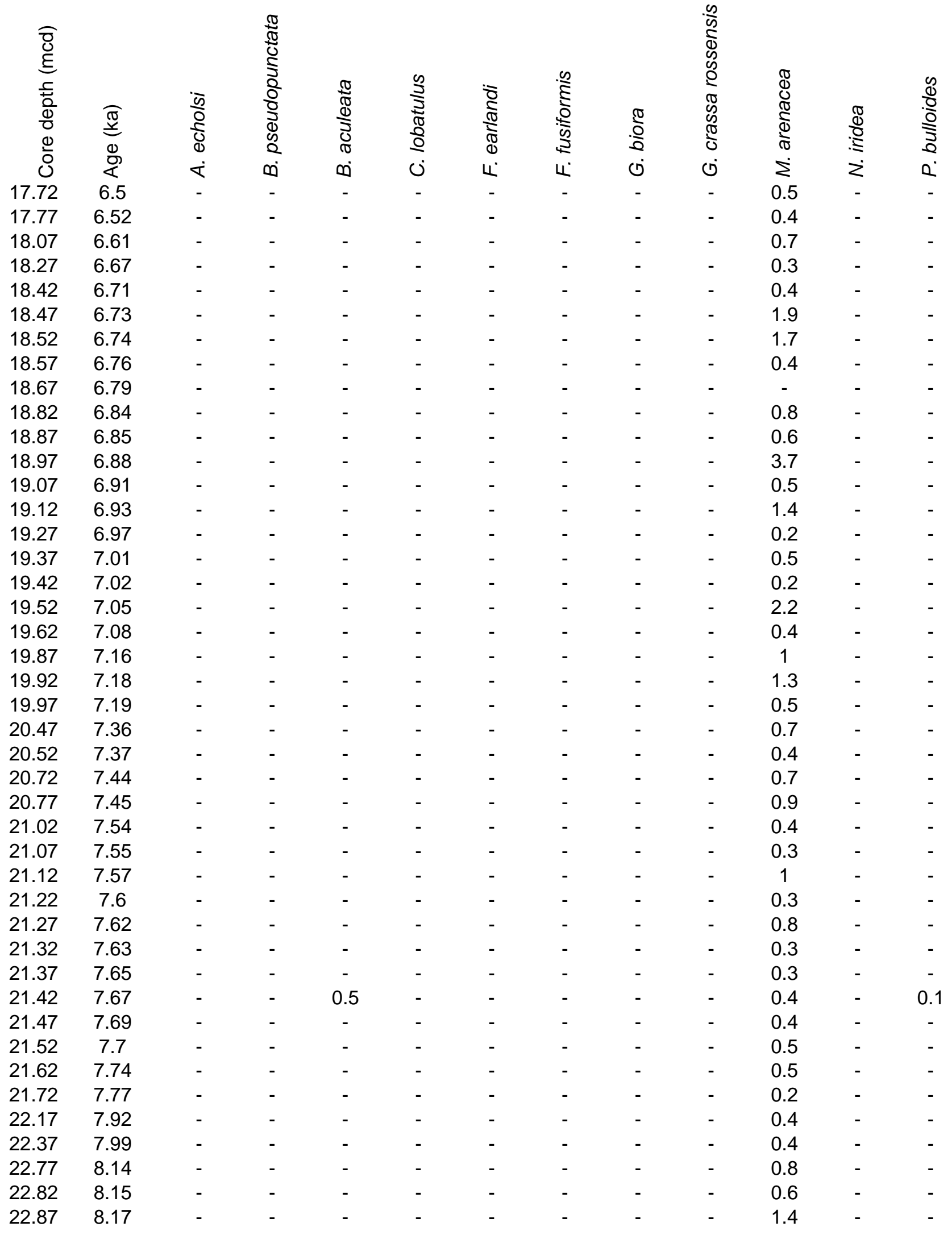


APPENDIX TABLE DR1: FORAMINIFERAL DISTRIBUTION DATA FOR CORE 1098B.

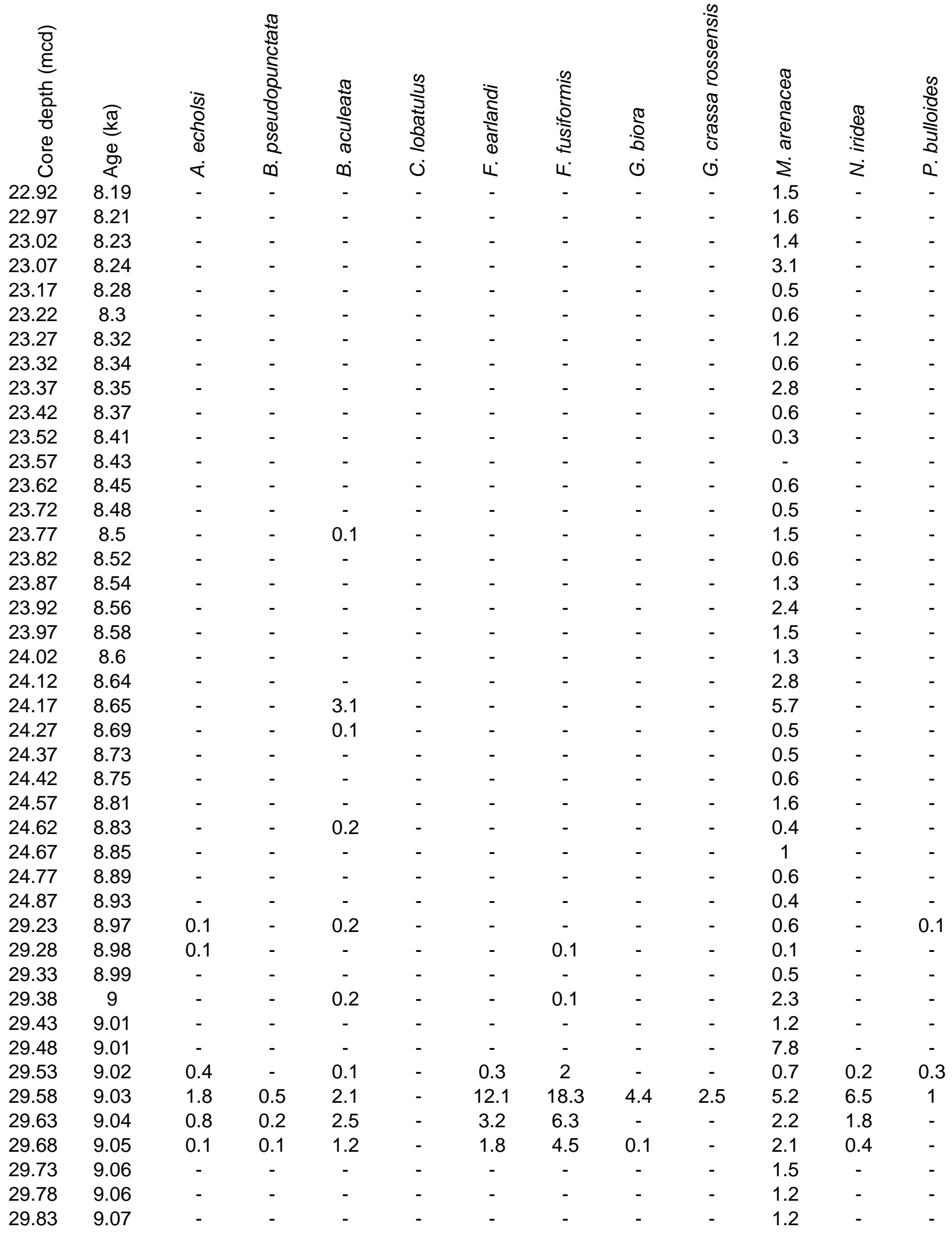


APPENDIX TABLE DR1: FORAMINIFERAL DISTRIBUTION DATA FOR CORE 1098B.

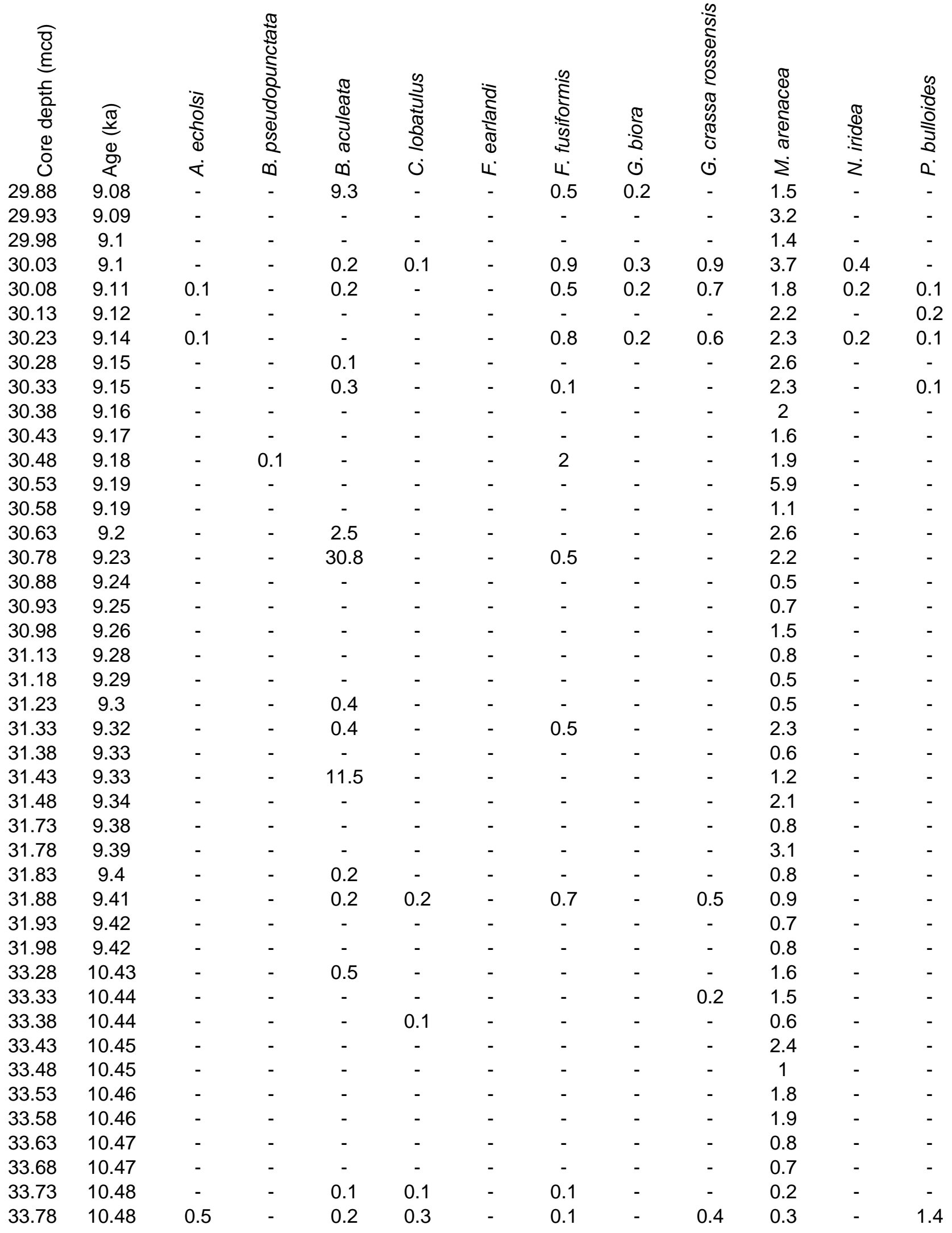


APPENDIX TABLE DR1: FORAMINIFERAL DISTRIBUTION DATA FOR CORE 1098B.

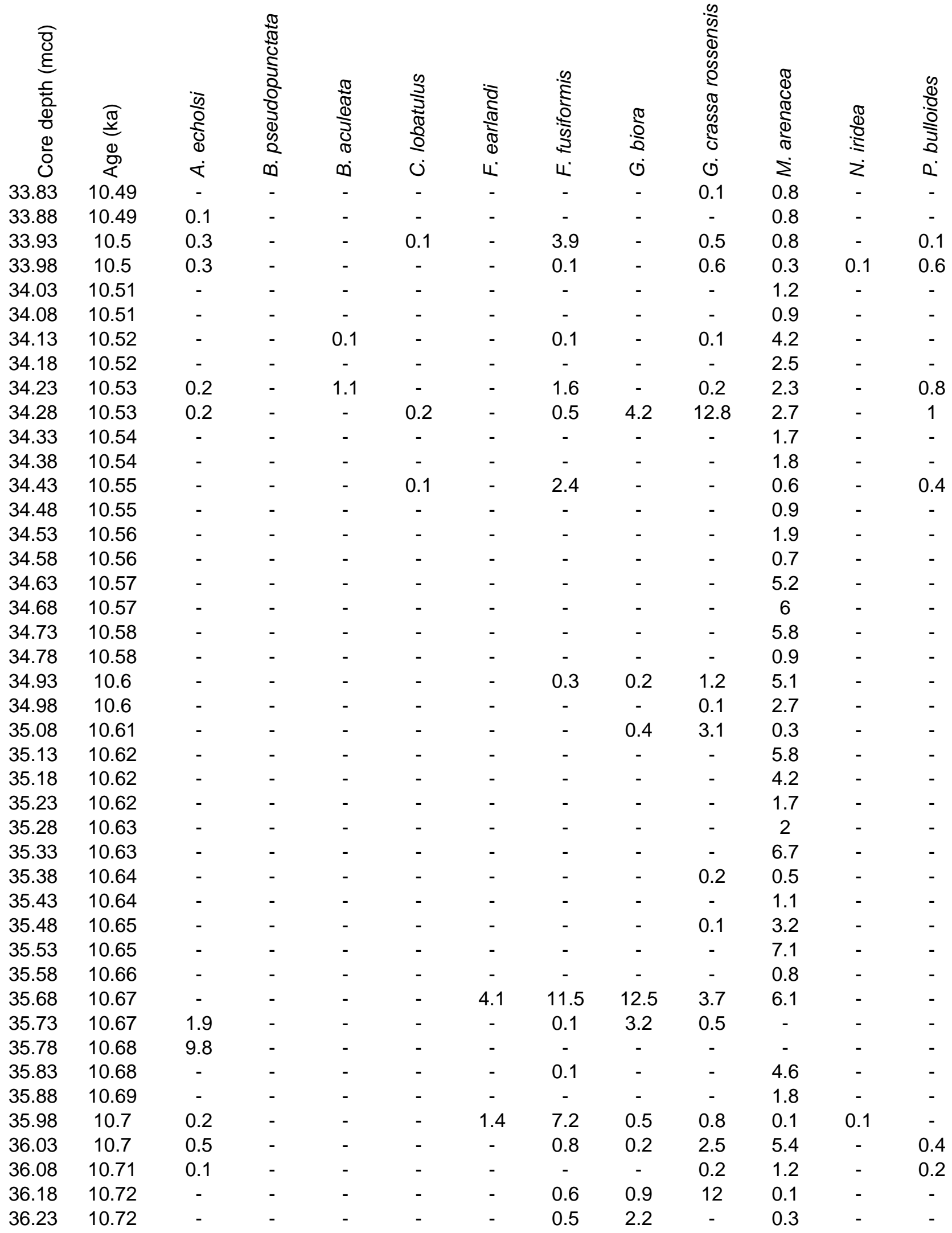


APPENDIX TABLE DR1: FORAMINIFERAL DISTRIBUTION DATA FOR CORE 1098B.

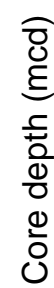

36.64

36.69

36.74

36.79

36.84

36.89

36.94

36.99

$37.04 \quad 10.8$

$37.09 \quad 10.81$

$37.14 \quad 10.81$

$\begin{array}{ll}37.19 & 10.82\end{array}$

$37.24 \quad 10.82$

$37.29 \quad 10.83$

$\begin{array}{ll}37.34 & 10.83\end{array}$

$37.39 \quad 10.84$

$37.44 \quad 10.84$

$37.49 \quad 10.85$

$37.54 \quad 10.85$

$37.59 \quad 10.86$

$37.64 \quad 10.86$

$37.69 \quad 10.87$

$\begin{array}{ll}37.74 & 10.87\end{array}$

$\begin{array}{ll}37.79 & 10.88\end{array}$

$37.84 \quad 10.88$

$37.89 \quad 10.89$

$37.94 \quad 10.89$

$37.99 \quad 10.9$

$38.04 \quad 10.9$

$38.09 \quad 10.91$

$38.14 \quad 10.91$

$38.19 \quad 10.92$

$38.24 \quad 10.92$

$38.29 \quad 10.92$

$38.39 \quad 10.93$

$38.44 \quad 10.94$

$38.49 \quad 10.94$

$38.54 \quad 10.95$

$38.59 \quad 10.95$

$38.64 \quad 10.96$

$38.69 \quad 10.96$

$38.74 \quad 10.97$

$38.79 \quad 10.97$
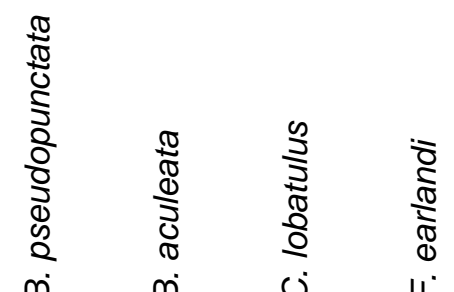

$0.1 \quad 0.1$

0.1

0.1

0.1

0.1

0.1

0.

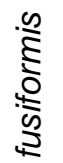

$\frac{9}{\frac{9}{2}}$

4

- -

0.1

-

$-$

2.3

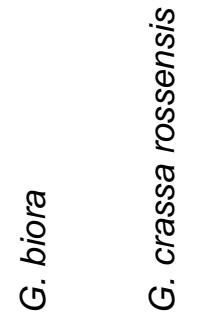

0.1

2.2

0.3

0.1
0.2

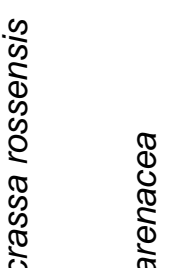

$-$

0.2

0.1

0.4

0.5

-

0.5
0.1

0.1

0.4

0.4
0.2

2.2

1.5

2.2

2.4

0.4

0.7

2.5

0.5

0.7

0.6

0.6

1.9

4.6

2.8

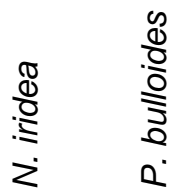

$\begin{array}{lll}4.7 & - & 0.1\end{array}$

3.5

1.5

2.9

0.5

2.5

4.7

$\begin{array}{llllll}0.1 & - & - & - & 0.2 & 1.6\end{array}$

$0.1 \quad-\quad 0.3$

0.1

$0.9 \quad 3.5$

0.1

0.3

0.6 \begin{tabular}{ll}
- & 0.3 \\
\hline
\end{tabular}

\begin{tabular}{ll}
- & 0.5 \\
\hline
\end{tabular}

$0.2 \quad 0.9$

$0.1 \quad 0.6$

0.1
1.2

2.2

2.8

2.7

5.3

0.1

0.8

0.7

4.7

2.2

0.5

2.4

0.8

5.5

8.6

3.6

0.8

$\begin{array}{ccccc}- & - & - & - & 0.8 \\ 0.2 & 1.9 & 1.4 & 0.1 & 0.5\end{array}$

0.2

.

$\begin{array}{llll}0.2 & 0.1 & - & 0.2\end{array}$

$\begin{array}{ll}- & 0.1 \\ 9.9 & 7.8\end{array}$

1.5

0.4

0.4

0.6

6.5

41.4

0.2

1.1

0.8

$0.2 \quad 0.1$

5.2

4.1 
APPENDIX TABLE DR1: FORAMINIFERAL DISTRIBUTION DATA FOR CORE 1098B.

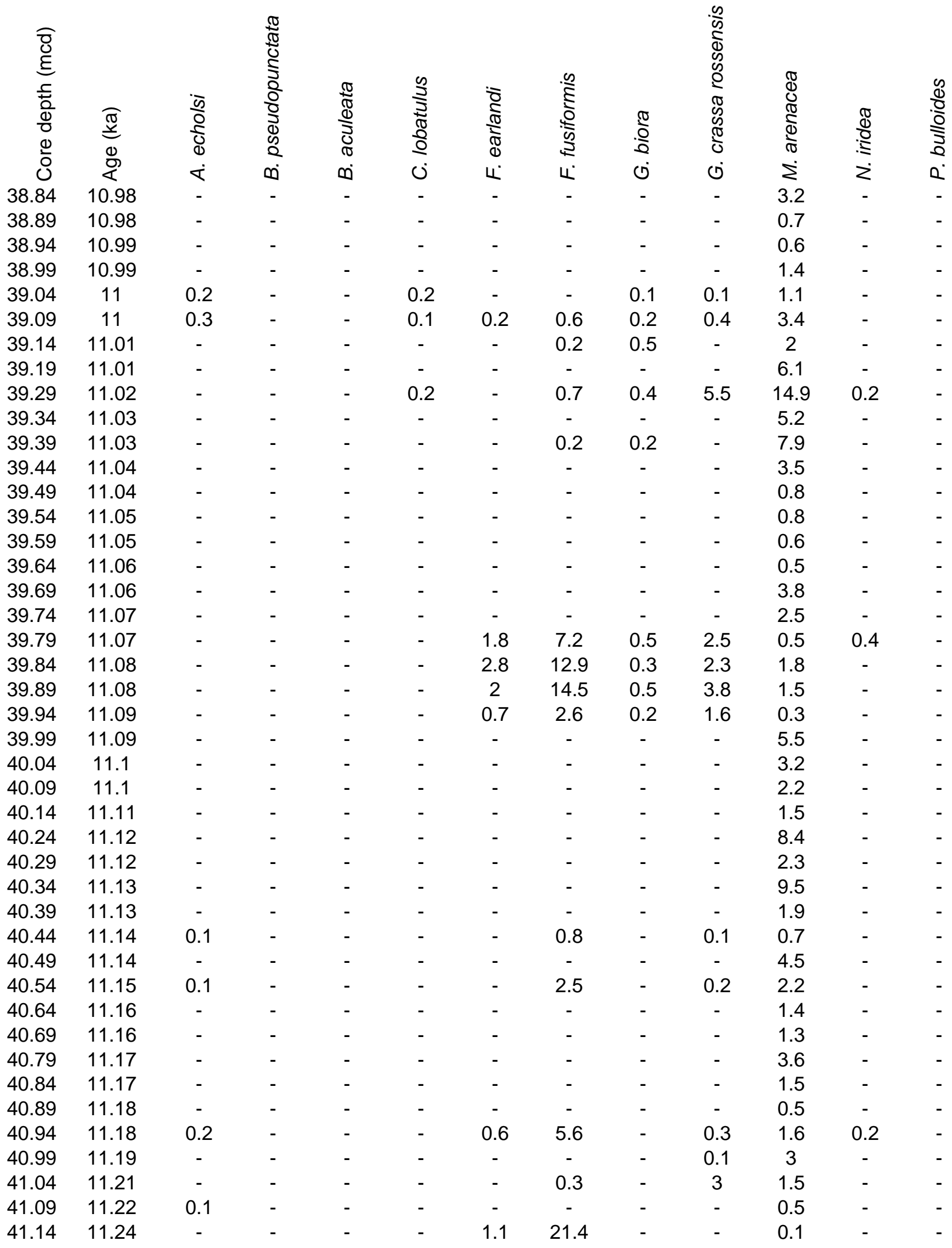


APPENDIX TABLE DR1: FORAMINIFERAL DISTRIBUTION DATA FOR CORE 1098B.

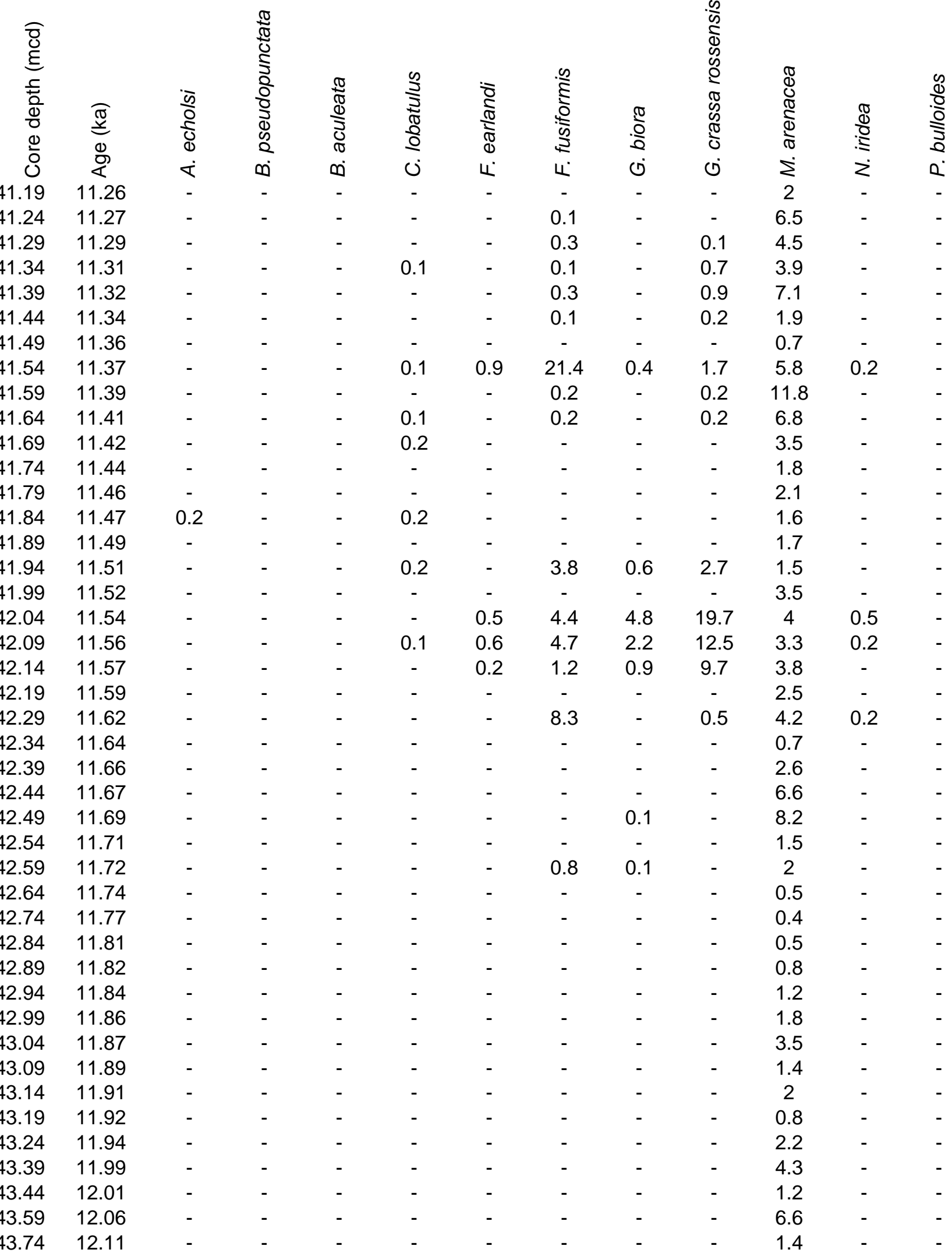


APPENDIX TABLE DR1: FORAMINIFERAL DISTRIBUTION DATA FOR CORE 1098B.

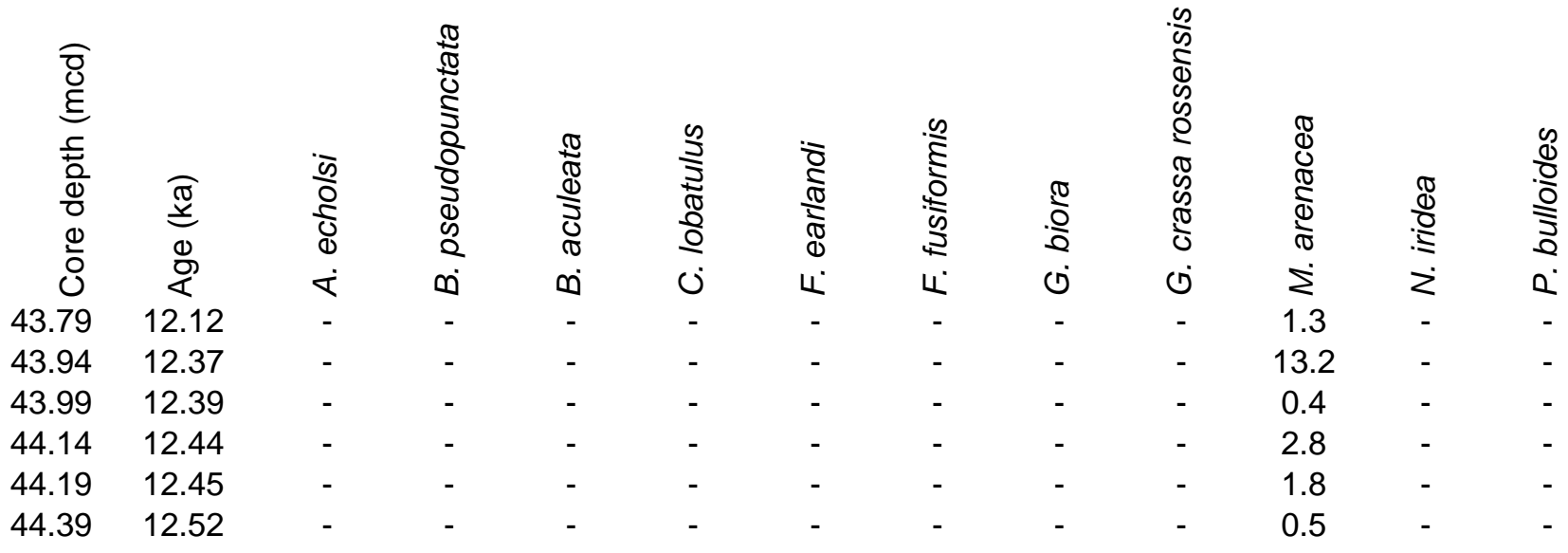




\section{APPENDIX TABLE}

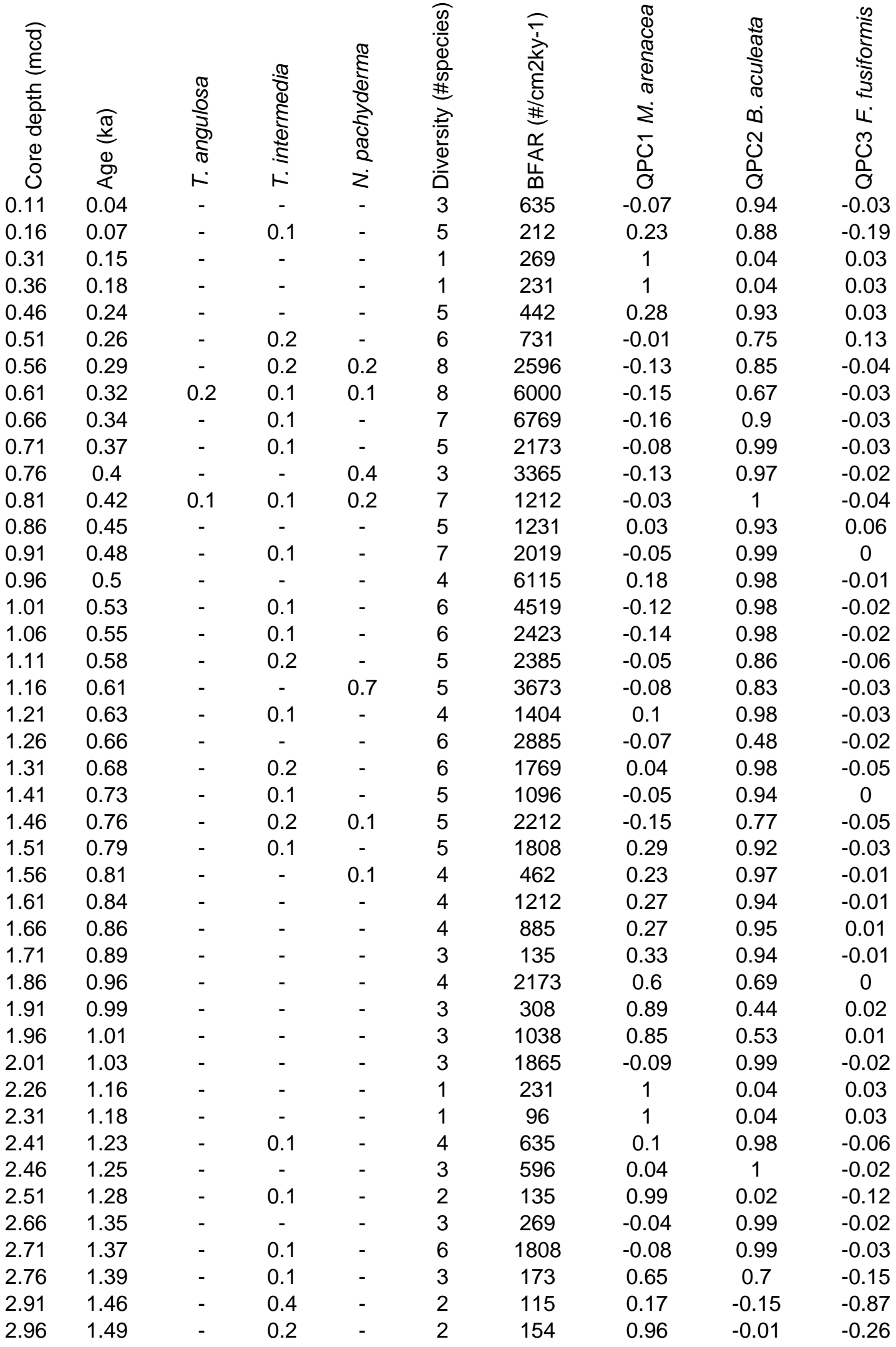




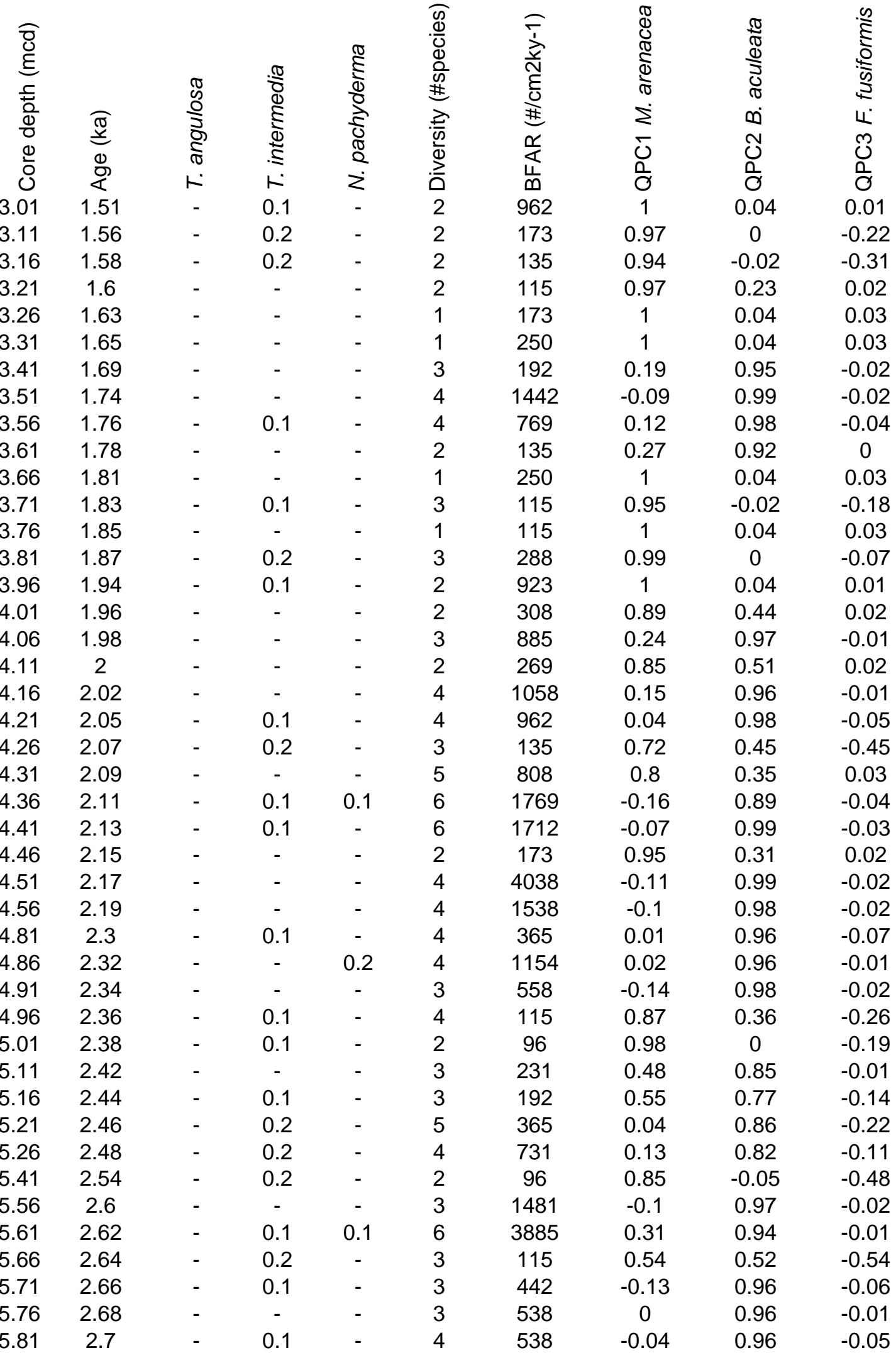




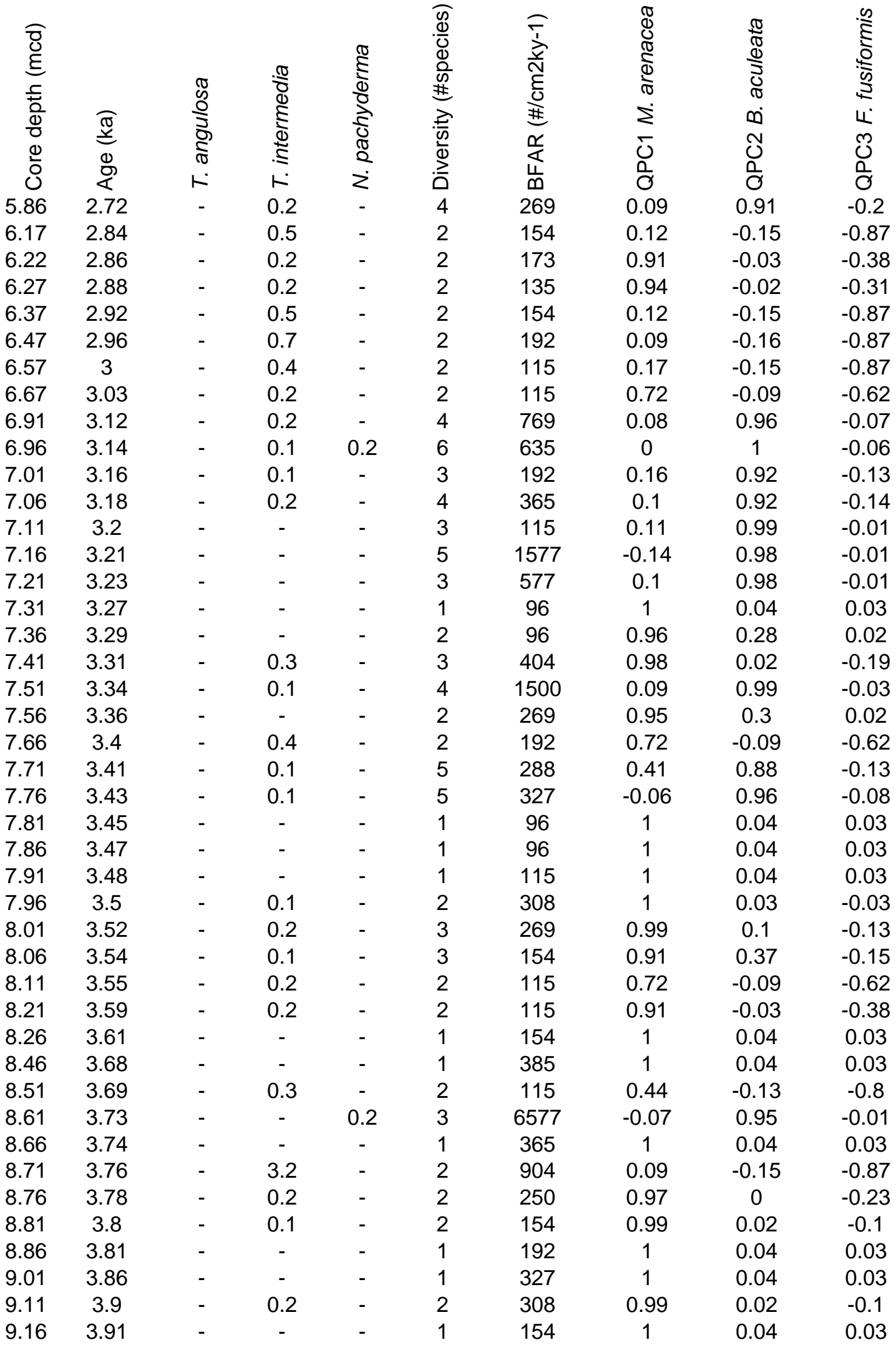




\begin{tabular}{|c|c|c|c|c|c|c|c|c|c|}
\hline 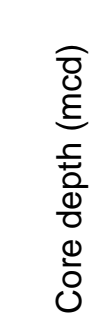 & 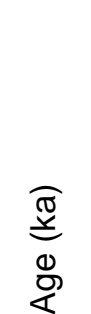 & 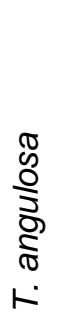 & 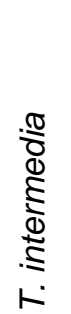 & 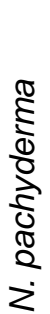 & 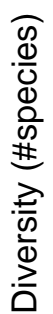 & 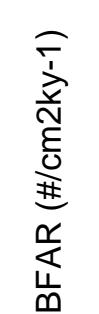 & 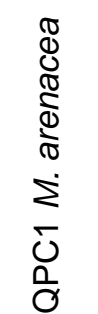 & 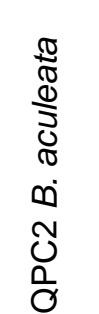 & 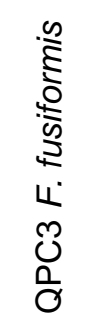 \\
\hline 9.21 & 3.93 & - & 0.1 & - & 2 & 250 & 1 & 0.03 & -0.05 \\
\hline 9.26 & 3.95 & - & 0.1 & - & 2 & 115 & 0.99 & 0.01 & -0.15 \\
\hline 9.31 & 3.96 & - & 0.2 & - & 2 & 115 & 0.91 & -0.03 & -0.38 \\
\hline 9.36 & 3.98 & - & - & - & 1 & 269 & 1 & 0.04 & 0.03 \\
\hline 9.41 & 4 & - & - & - & 1 & 192 & 1 & 0.04 & 0.03 \\
\hline 9.51 & 4.03 & - & 0.1 & - & 2 & 96 & 0.98 & 0 & -0.19 \\
\hline 9.56 & 4.05 & - & 0.2 & - & 2 & 135 & 0.94 & -0.02 & -0.31 \\
\hline 9.66 & 4.08 & - & 0.2 & - & 2 & 692 & 1 & 0.03 & -0.05 \\
\hline 9.71 & 4.09 & - & 0.2 & - & 2 & 269 & 0.99 & 0.02 & -0.12 \\
\hline 10.06 & 4.21 & - & 0.1 & - & 2 & 212 & 1 & 0.03 & -0.06 \\
\hline 10.16 & 4.24 & - & 0.1 & - & 2 & 2019 & 1 & 0.04 & 0.02 \\
\hline 10.21 & 4.26 & - & - & - & 1 & 346 & 1 & 0.04 & 0.03 \\
\hline 10.26 & 4.27 & - & - & - & 1 & 135 & 1 & 0.04 & 0.03 \\
\hline 10.31 & 4.29 & - & 0.3 & - & 2 & 1462 & 1 & 0.03 & -0.02 \\
\hline 10.36 & 4.3 & - & - & - & 1 & 192 & 1 & 0.04 & 0.03 \\
\hline 10.51 & 4.35 & - & 0.1 & - & 2 & 135 & 0.99 & 0.02 & -0.12 \\
\hline 10.56 & 4.37 & - & - & - & 2 & 154 & 0.98 & 0.07 & 0.02 \\
\hline 10.61 & 4.38 & - & 0.1 & - & 2 & 154 & 0.99 & 0.02 & -0.1 \\
\hline 10.86 & 4.46 & - & 0.2 & - & 3 & 288 & 0.91 & 0.06 & -0.26 \\
\hline 10.91 & 4.48 & - & 0.2 & - & 2 & 173 & 0.97 & 0 & -0.22 \\
\hline 10.96 & 4.49 & - & 0.3 & - & 3 & 250 & 0.77 & 0.34 & -0.47 \\
\hline 11.01 & 4.51 & - & 0.1 & - & 2 & 250 & 1 & 0.03 & -0.05 \\
\hline 11.06 & 4.52 & - & - & - & 1 & 231 & 1 & 0.04 & 0.03 \\
\hline 11.11 & 4.54 & - & 0.2 & - & 2 & 288 & 0.99 & 0.02 & -0.11 \\
\hline 11.16 & 4.56 & - & - & - & 1 & 135 & 1 & 0.04 & 0.03 \\
\hline 11.21 & 4.57 & - & 0.1 & - & 2 & 115 & 0.99 & 0.01 & -0.15 \\
\hline 11.26 & 4.59 & - & 0.2 & - & 2 & 115 & 0.72 & -0.09 & -0.62 \\
\hline 11.31 & 4.6 & - & - & - & 1 & 212 & 1 & 0.04 & 0.03 \\
\hline 11.41 & 4.63 & - & 0.2 & - & 2 & 173 & 0.97 & 0 & -0.22 \\
\hline 11.46 & 4.65 & - & 0.2 & - & 2 & 96 & 0.85 & -0.05 & -0.48 \\
\hline 11.51 & 4.66 & - & 2.7 & - & 2 & 731 & 0.06 & -0.16 & -0.87 \\
\hline 11.56 & 4.68 & - & 0.5 & - & 2 & 173 & 0.44 & -0.13 & -0.8 \\
\hline 11.61 & 4.69 & - & 0.1 & - & 2 & 96 & 0.98 & 0 & -0.19 \\
\hline 11.71 & 4.73 & - & 0.2 & - & 4 & 231 & 0.34 & 0.32 & -0.28 \\
\hline 11.81 & 4.76 & - & 0.1 & - & 2 & 1692 & 1 & 0.04 & 0.02 \\
\hline 11.86 & 4.77 & - & 0.2 & - & 2 & 500 & 1 & 0.03 & -0.05 \\
\hline 11.96 & 4.8 & - & 0.1 & - & 2 & 1442 & 1 & 0.04 & 0.02 \\
\hline 12.01 & 4.82 & - & - & - & 1 & 154 & 1 & 0.04 & 0.03 \\
\hline 12.06 & 4.83 & - & - & - & 1 & 615 & 1 & 0.04 & 0.03 \\
\hline 12.11 & 4.85 & - & 0.1 & - & 2 & 481 & 1 & 0.04 & -0.01 \\
\hline 12.16 & 4.86 & - & - & - & 2 & 692 & -0.09 & 0.94 & -0.01 \\
\hline 12.56 & 4.98 & - & - & - & 1 & 692 & 1 & 0.04 & 0.03 \\
\hline 13.01 & 5.12 & - & - & - & 1 & 481 & 1 & 0.04 & 0.03 \\
\hline
\end{tabular}




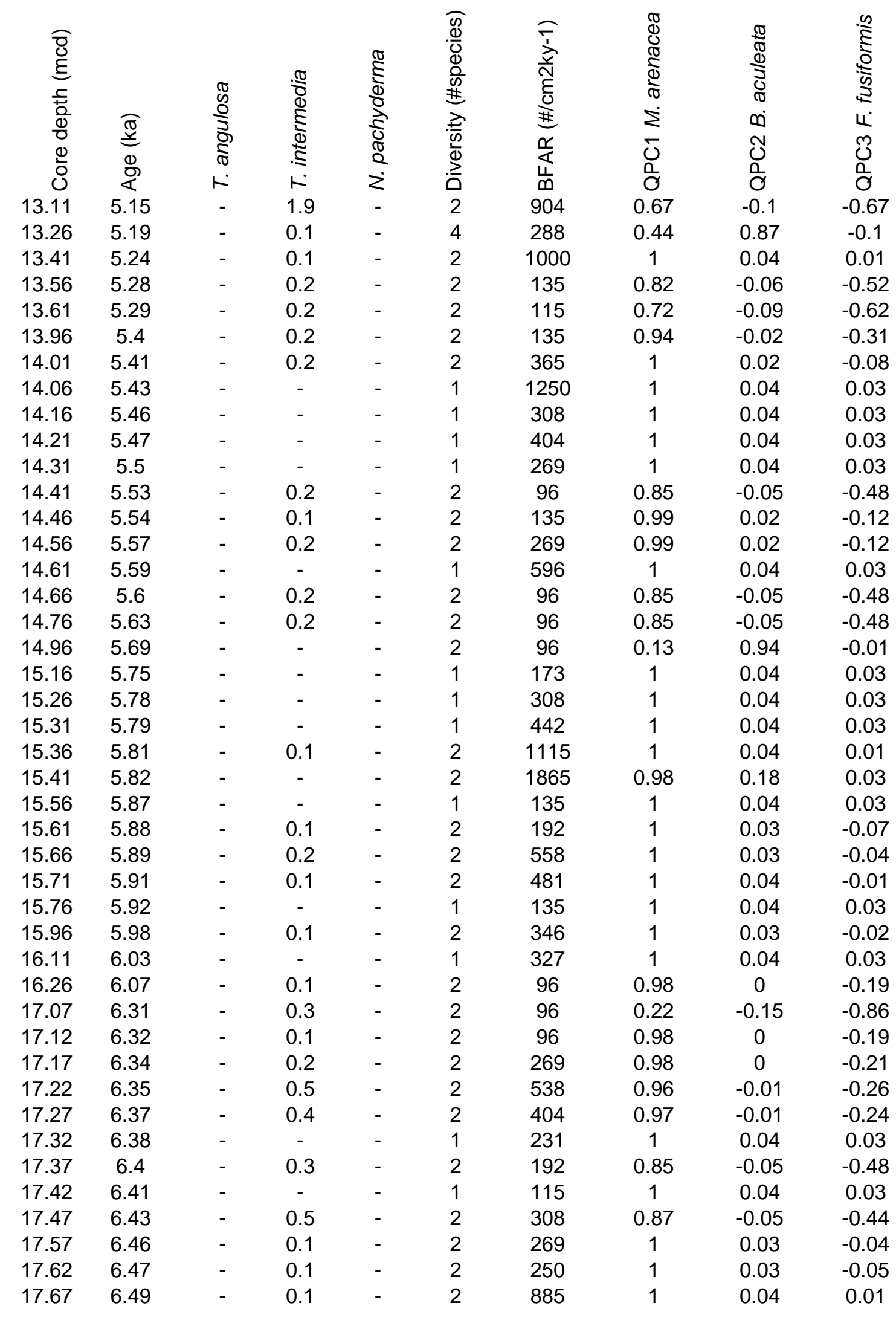




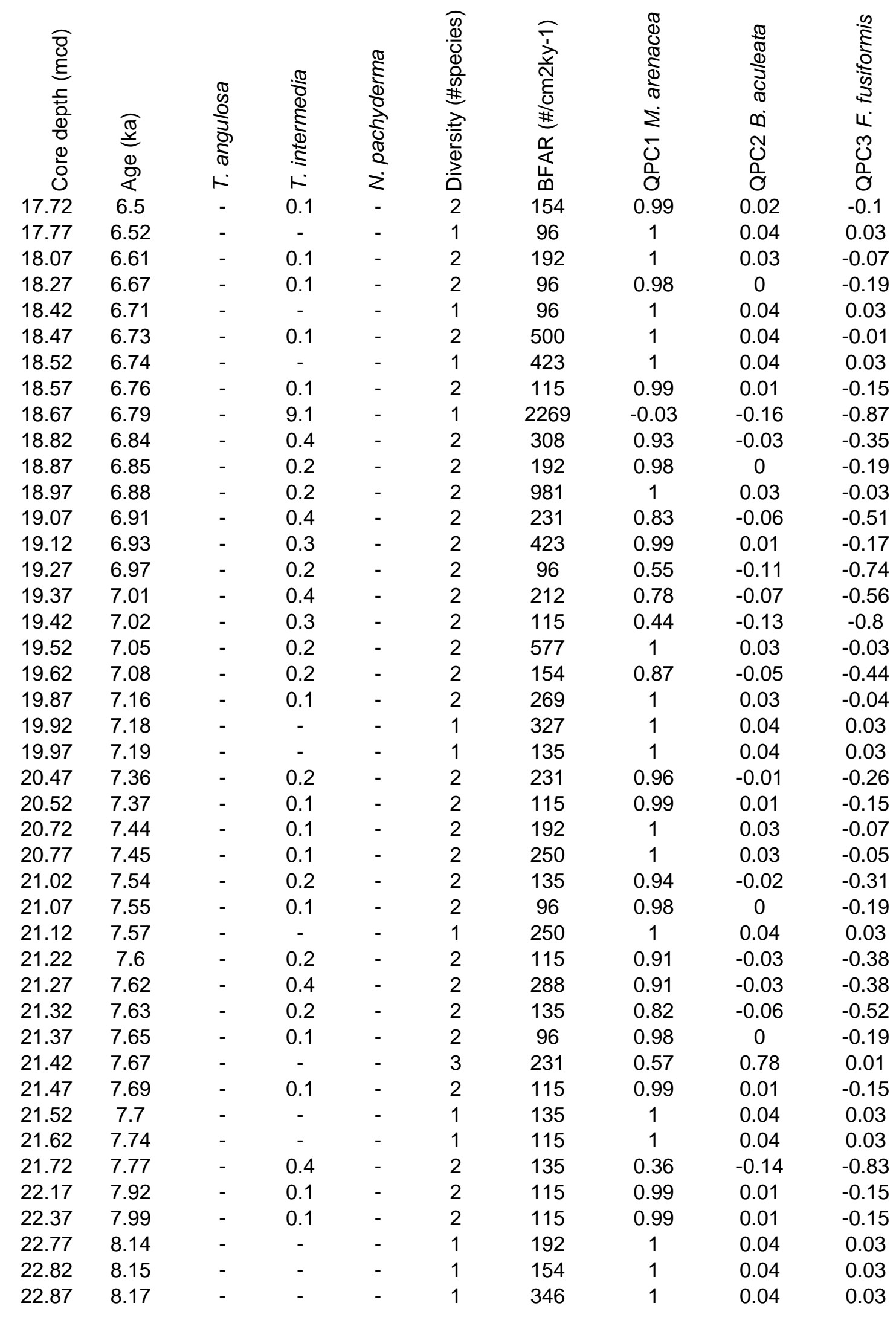




\begin{tabular}{|c|c|c|c|c|c|c|c|c|c|}
\hline 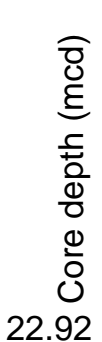 & 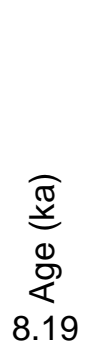 & 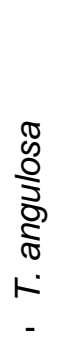 & 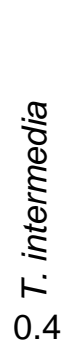 & 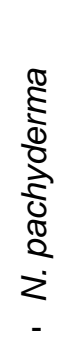 & 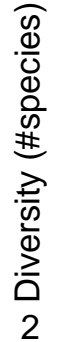 & 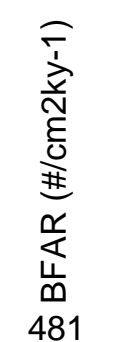 & 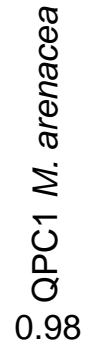 & 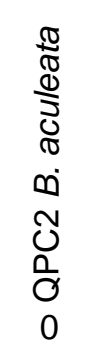 & 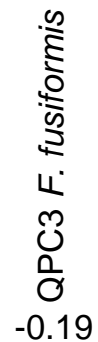 \\
\hline 22.97 & 8.21 & - & - & - & 1 & 404 & 1 & 0.04 & 0.03 \\
\hline 23.02 & 8.23 & - & - & - & 1 & 346 & 1 & 0.04 & 0.03 \\
\hline 23.07 & 8.24 & - & 0.2 & - & 2 & 827 & 1 & 0.03 & -0.04 \\
\hline 23.17 & 8.28 & - & 0.1 & - & 2 & 154 & 0.99 & 0.02 & -0.1 \\
\hline 23.22 & 8.3 & - & - & - & 1 & 154 & 1 & 0.04 & 0.03 \\
\hline 23.27 & 8.32 & - & - & - & 1 & 288 & 1 & 0.04 & 0.03 \\
\hline 23.32 & 8.34 & - & - & - & 1 & 154 & 1 & 0.04 & 0.03 \\
\hline 23.37 & 8.35 & - & 0.2 & - & 2 & 731 & 1 & 0.03 & -0.02 \\
\hline 23.42 & 8.37 & - & 0.2 & - & 2 & 192 & 0.98 & 0 & -0.19 \\
\hline 23.52 & 8.41 & - & 0.1 & - & 2 & 96 & 0.98 & 0 & -0.19 \\
\hline 23.57 & 8.43 & - & 0.5 & - & 1 & 135 & -0.03 & -0.16 & -0.87 \\
\hline 23.62 & 8.45 & - & 0.1 & - & 2 & 173 & 1 & 0.02 & -0.08 \\
\hline 23.72 & 8.48 & - & - & - & 1 & 115 & 1 & 0.04 & 0.03 \\
\hline 23.77 & 8.5 & - & 0.3 & - & 3 & 481 & 0.99 & 0.06 & -0.15 \\
\hline 23.82 & 8.52 & - & 0.2 & - & 2 & 192 & 0.98 & 0 & -0.19 \\
\hline 23.87 & 8.54 & - & - & - & 1 & 327 & 1 & 0.04 & 0.03 \\
\hline 23.92 & 8.56 & - & - & - & 1 & 596 & 1 & 0.04 & 0.03 \\
\hline 23.97 & 8.58 & - & - & - & 1 & 365 & 1 & 0.04 & 0.03 \\
\hline 24.02 & 8.6 & - & - & - & 1 & 327 & 1 & 0.04 & 0.03 \\
\hline 24.12 & 8.64 & - & - & - & 1 & 692 & 1 & 0.04 & 0.03 \\
\hline 24.17 & 8.65 & - & 0.1 & - & 3 & 2212 & 0.85 & 0.5 & 0.01 \\
\hline 24.27 & 8.69 & - & 0.2 & - & 3 & 422 & 0.95 & 0.14 & -0.26 \\
\hline 24.37 & 8.73 & - & - & - & 1 & 329 & 1 & 0.04 & 0.03 \\
\hline 24.42 & 8.75 & - & - & - & 1 & 375 & 1 & 0.04 & 0.03 \\
\hline 24.57 & 8.81 & - & 0.2 & - & 2 & 1126 & 0.99 & 0.02 & -0.1 \\
\hline 24.62 & 8.83 & - & - & - & 2 & 329 & 0.91 & 0.4 & 0.02 \\
\hline 24.67 & 8.85 & - & - & - & 1 & 610 & 1 & 0.04 & 0.03 \\
\hline 24.77 & 8.89 & - & - & - & 1 & 375 & 1 & 0.04 & 0.03 \\
\hline 24.87 & 8.93 & - & - & - & 1 & 235 & 1 & 0.04 & 0.03 \\
\hline 29.23 & 8.97 & - & - & - & 4 & 563 & 0.95 & 0.25 & 0.03 \\
\hline 29.28 & 8.98 & - & 0.2 & 0.1 & 4 & 282 & 0.35 & -0.23 & -0.5 \\
\hline 29.33 & 8.99 & - & - & - & 1 & 329 & 1 & 0.04 & 0.03 \\
\hline 29.38 & 9 & - & - & 0.3 & 3 & 1737 & 0.99 & 0.1 & 0.04 \\
\hline 29.43 & 9.01 & - & - & - & 1 & 704 & 1 & 0.04 & 0.03 \\
\hline 29.48 & 9.01 & - & 0.2 & - & 2 & 4881 & 1 & 0.04 & 0.01 \\
\hline 29.53 & 9.02 & - & - & 0.2 & 7 & 2534 & 0.24 & -0.16 & 0.56 \\
\hline 29.58 & 9.03 & - & 0.2 & 2.6 & 11 & 34777 & 0.08 & -0.18 & 0.58 \\
\hline 29.63 & 9.04 & - & - & 0.8 & 7 & 10795 & 0.15 & 0.13 & 0.53 \\
\hline 29.68 & 9.05 & - & - & 0.2 & 8 & 6430 & 0.3 & 0.07 & 0.53 \\
\hline 29.73 & 9.06 & - & - & - & 1 & 892 & 1 & 0.04 & 0.03 \\
\hline 29.78 & 9.06 & - & - & - & 1 & 751 & 1 & 0.04 & 0.03 \\
\hline 29.83 & 9.07 & - & - & - & 1 & 704 & 1 & 0.04 & 0.03 \\
\hline
\end{tabular}




\begin{tabular}{|c|c|c|c|c|c|c|c|c|c|}
\hline 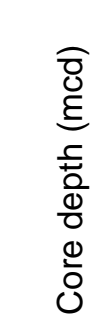 & 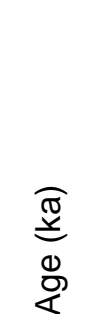 & 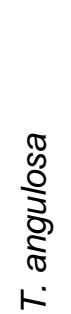 & 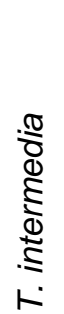 & 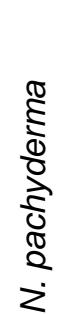 & 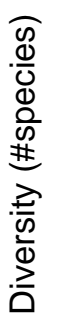 & 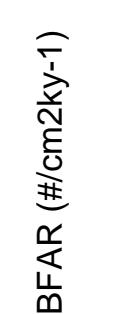 & 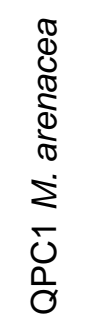 & 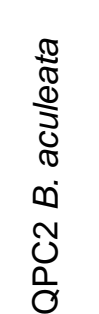 & 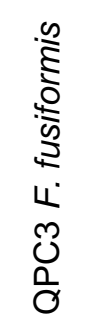 \\
\hline 29.88 & 9.08 & - & - & - & 4 & 6946 & 0.04 & 0.94 & 0.02 \\
\hline 29.93 & 9.09 & - & - & - & 1 & 1924 & 1 & 0.04 & 0.03 \\
\hline 29.98 & 9.1 & - & - & - & 1 & 845 & 1 & 0.04 & 0.03 \\
\hline 30.03 & 9.1 & - & 0.1 & 0.1 & 8 & 4083 & 0.95 & 0 & 0.19 \\
\hline 30.08 & 9.11 & - & - & - & 8 & 2300 & 0.91 & -0.01 & 0.25 \\
\hline 30.13 & 9.12 & - & - & - & 2 & 1408 & 0.99 & 0.04 & 0.03 \\
\hline 30.23 & 9.14 & - & - & 0.2 & 7 & 2628 & 0.93 & -0.06 & 0.25 \\
\hline 30.28 & 9.15 & - & - & - & 2 & 1643 & 1 & 0.07 & 0.03 \\
\hline 30.33 & 9.15 & - & - & 0.2 & 4 & 1830 & 0.98 & 0.16 & 0.04 \\
\hline 30.38 & 9.16 & - & - & - & 1 & 1220 & 1 & 0.04 & 0.03 \\
\hline 30.43 & 9.17 & - & - & - & 1 & 986 & 1 & 0.04 & 0.03 \\
\hline 30.48 & 9.18 & - & - & - & 3 & 2441 & 0.68 & -0.07 & 0.42 \\
\hline 30.53 & 9.19 & - & - & - & 1 & 3614 & 1 & 0.04 & 0.03 \\
\hline 30.58 & 9.19 & - & - & - & 1 & 657 & 1 & 0.04 & 0.03 \\
\hline 30.63 & 9.2 & 0.1 & 0.1 & - & 4 & 3191 & 0.68 & 0.7 & -0.01 \\
\hline 30.78 & 9.23 & - & 0.1 & - & 4 & 20416 & -0.05 & 0.95 & -0.01 \\
\hline 30.88 & 9.24 & - & - & - & 1 & 329 & 1 & 0.04 & 0.03 \\
\hline 30.93 & 9.25 & - & - & - & 1 & 422 & 1 & 0.04 & 0.03 \\
\hline 30.98 & 9.26 & - & - & - & 1 & 892 & 1 & 0.04 & 0.03 \\
\hline 31.13 & 9.28 & - & - & - & 1 & 469 & 1 & 0.04 & 0.03 \\
\hline 31.18 & 9.29 & - & - & - & 1 & 329 & 1 & 0.04 & 0.03 \\
\hline 31.23 & 9.3 & - & - & - & 2 & 563 & 0.78 & 0.61 & 0.01 \\
\hline 31.33 & 9.32 & - & - & - & 3 & 1924 & 0.97 & 0.17 & 0.13 \\
\hline 31.38 & 9.33 & - & - & - & 1 & 375 & 1 & 0.04 & 0.03 \\
\hline 31.43 & 9.33 & - & - & - & 2 & 7744 & -0.02 & 0.95 & -0.01 \\
\hline 31.48 & 9.34 & - & - & - & 1 & 1267 & 1 & 0.04 & 0.03 \\
\hline 31.73 & 9.38 & - & 0.2 & - & 2 & 563 & 0.99 & 0.01 & -0.15 \\
\hline 31.78 & 9.39 & - & 0.1 & - & 2 & 1924 & 1 & 0.04 & 0.01 \\
\hline 31.83 & 9.4 & - & 0.1 & - & 3 & 610 & 0.97 & 0.22 & -0.06 \\
\hline 31.88 & 9.41 & 0.1 & - & 0.2 & 6 & 1737 & 0.72 & 0.05 & 0.42 \\
\hline 31.93 & 9.42 & - & - & - & 1 & 422 & 1 & 0.04 & 0.03 \\
\hline 31.98 & 9.42 & - & - & - & 1 & 516 & 1 & 0.04 & 0.03 \\
\hline 33.28 & 10.43 & - & - & - & 2 & 2198 & 0.93 & 0.35 & 0.02 \\
\hline 33.33 & 10.44 & - & - & - & 2 & 1805 & 0.99 & 0.02 & 0.05 \\
\hline 33.38 & 10.44 & - & - & - & 2 & 706 & 0.99 & 0.03 & 0.03 \\
\hline 33.43 & 10.45 & - & - & - & 1 & 2433 & 1 & 0.04 & 0.03 \\
\hline 33.48 & 10.45 & - & - & - & 1 & 1020 & 1 & 0.04 & 0.03 \\
\hline 33.53 & 10.46 & - & - & - & 1 & 1884 & 1 & 0.04 & 0.03 \\
\hline 33.58 & 10.46 & - & - & - & 1 & 1962 & 1 & 0.04 & 0.03 \\
\hline 33.63 & 10.47 & - & - & - & 1 & 785 & 1 & 0.04 & 0.03 \\
\hline 33.68 & 10.47 & - & - & - & 1 & 706 & 1 & 0.04 & 0.03 \\
\hline 33.73 & 10.48 & 0.1 & - & 0.1 & 5 & 628 & 0.84 & 0.23 & 0.19 \\
\hline 33.78 & 10.48 & 0.1 & - & 0.4 & 8 & 3689 & 0.04 & -0.03 & 0.1 \\
\hline
\end{tabular}




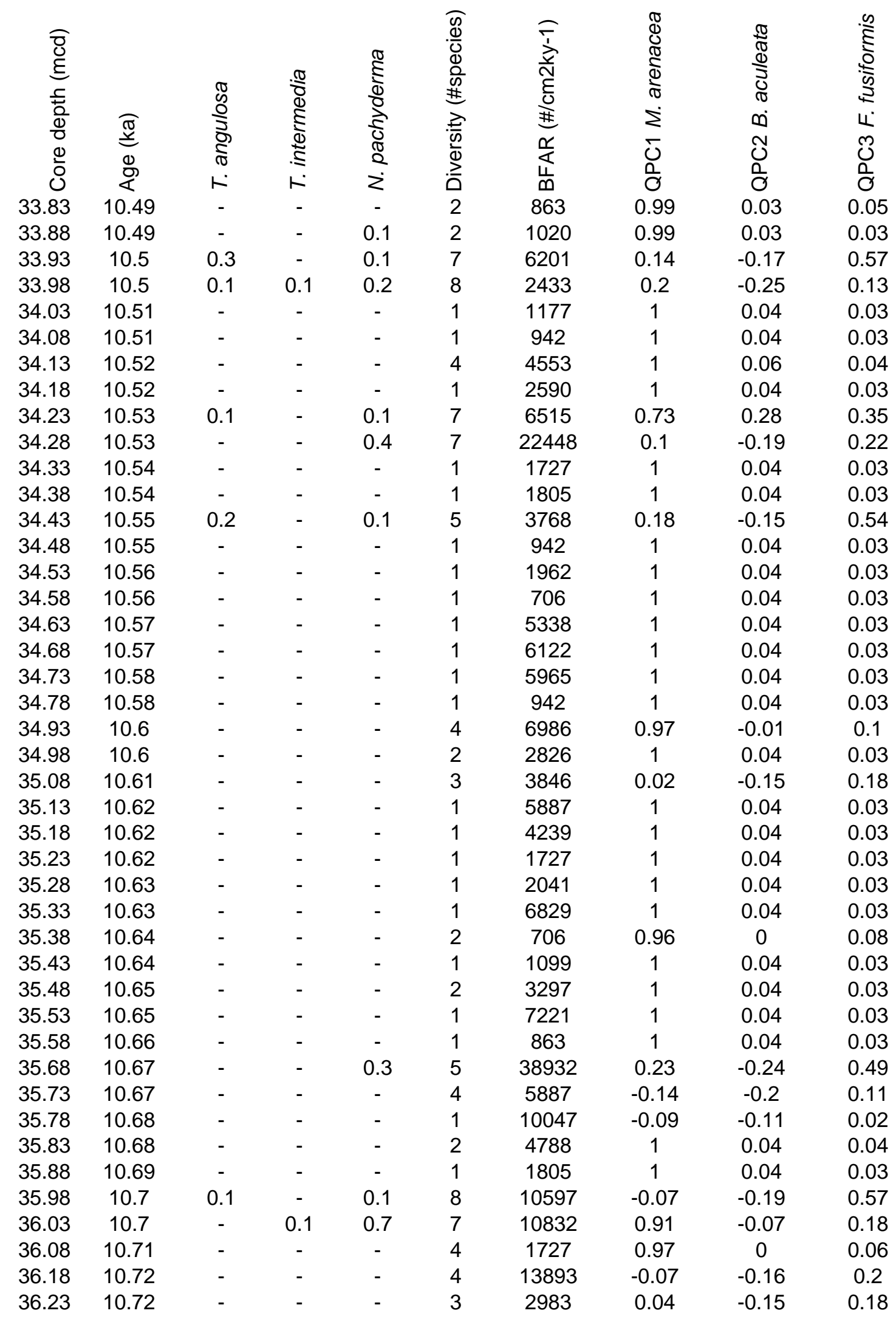




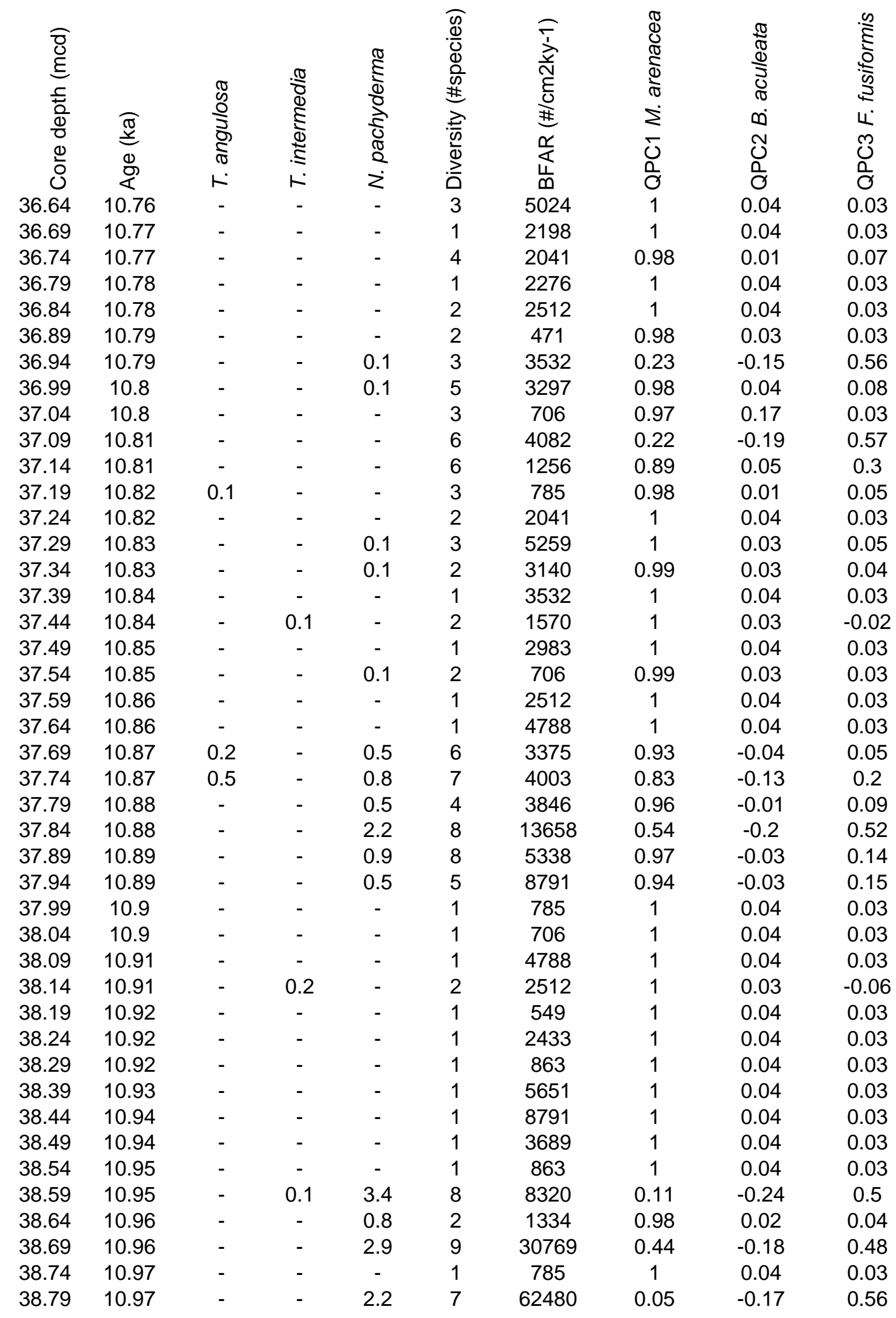




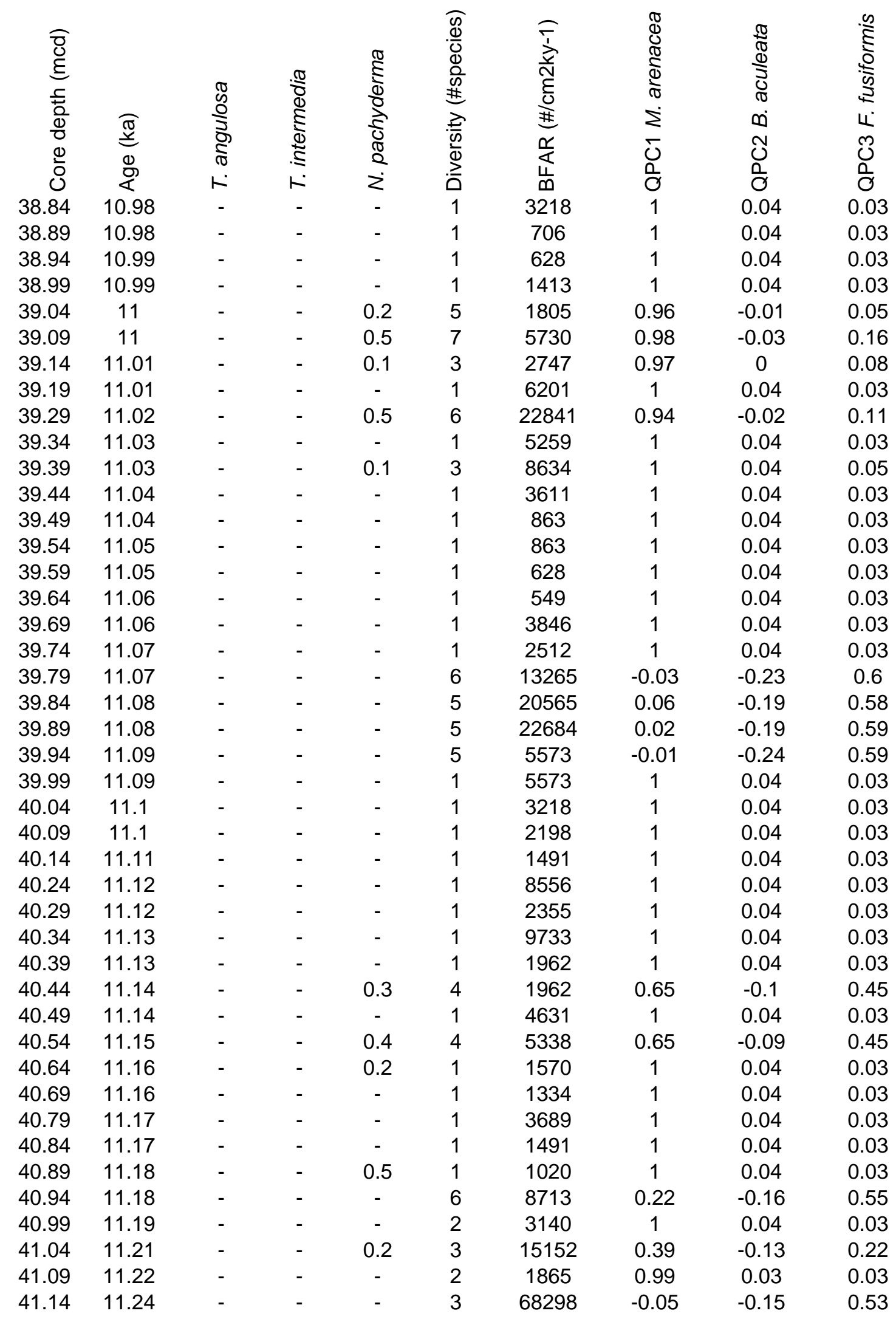




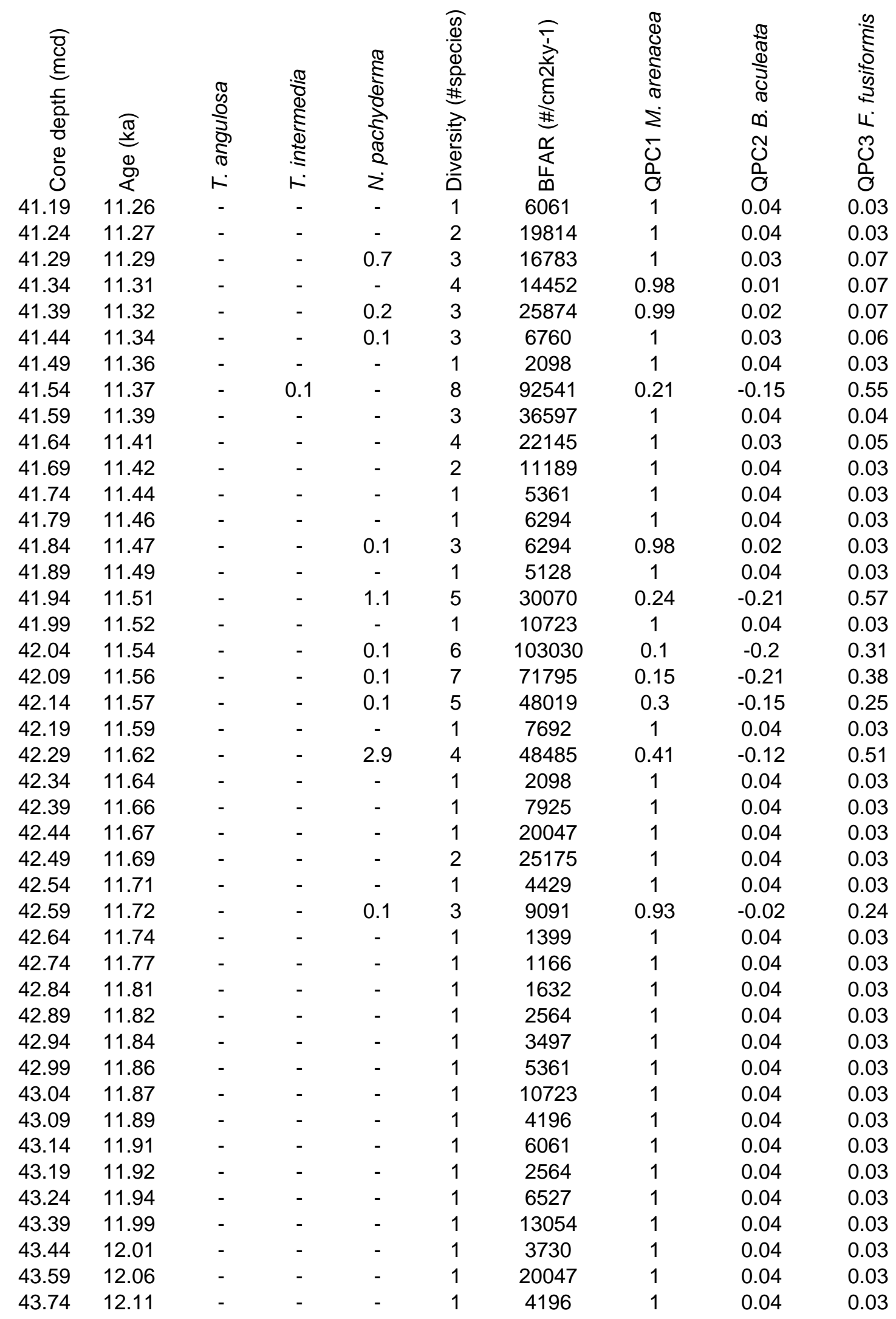




\section{APPENDIX TABLE}

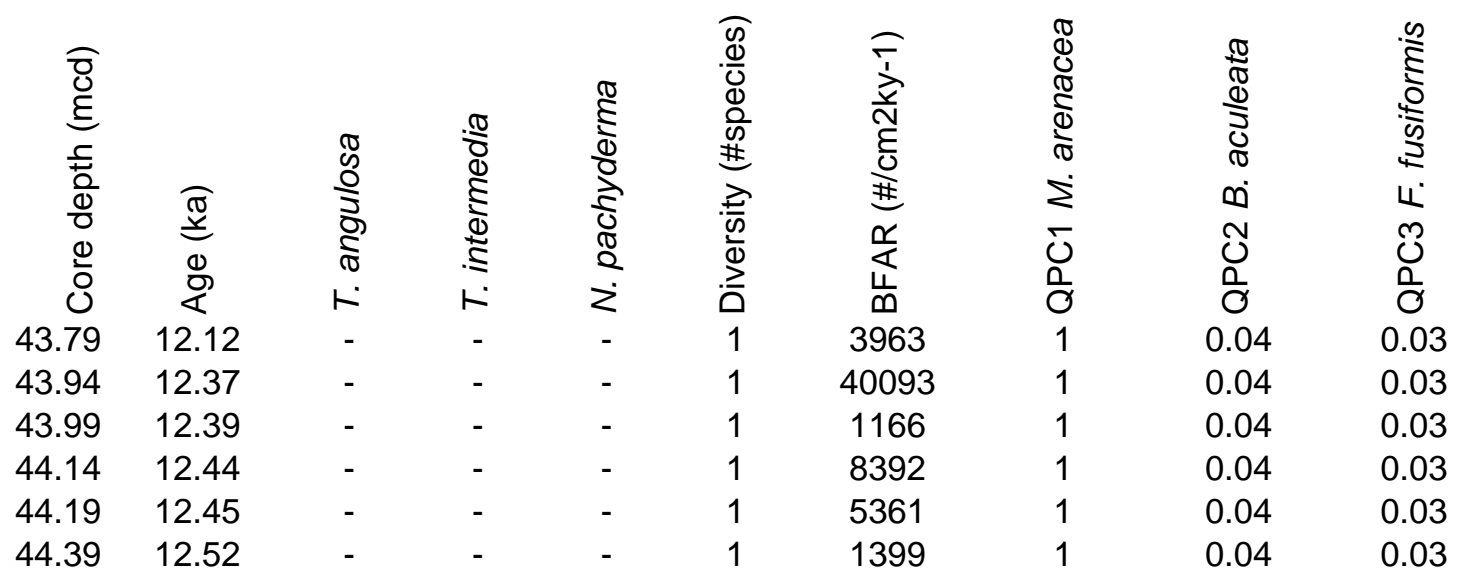


APPENDIX TABLE DR2: R-MODE PRINCIPAL COMPONENT SCORES FOR CORE 1098B.

\begin{tabular}{lccc}
\hline \hline Species & PC1 & PC2 & PC3 \\
\hline Astrononion echolsi & -0.288 & -0.368 & 0.076 \\
Bolivina pseudopunctata & -0.356 & 0.721 & -0.088 \\
Bulimina aculeata & -0.385 & 3.133 & -0.050 \\
Cibicides lobatulus & -0.307 & -0.357 & -0.001 \\
Fursenkoina earlandi & -0.304 & -0.373 & 0.210 \\
Fursenkoina fusiformis & -0.164 & -0.472 & 1.758 \\
Globocassidulina biora & -0.292 & -0.413 & 0.219 \\
Globocassidulina crassa rossensis & -0.226 & -0.471 & 0.569 \\
Miliammina arenacea & 3.318 & 0.144 & 0.093 \\
Nonionella iridea & -0.307 & -0.370 & 0.081 \\
Pullenia bulloides & -0.295 & -0.282 & 0.043 \\
Trochammina intermedia & -0.085 & -0.531 & -2.909 \\
Trifarina angulosa & -0.308 & -0.360 & -0.001 \\
\hline variance explained $(\%)$ & 72.49 & 13.66 & 5.63 \\
\hline
\end{tabular}

